\title{
Design of computerized controlled systems for directional laying of engineering communications
}

\author{
Sergey Menshenin*, Elena Menshenina \\ Sedov Water Transport Institute, branch of Admiral Ushakov State Maritime University, 8, Sedova st., Rostov-on-Don, 344006, \\ Russia
}

\begin{abstract}
The subject of research and development is the technology of directional laying of engineering horizontal inclined wells intended both for exploration drilling and for laying engineering communications by the method of static puncture, as well as installations for their implementation. Laying engineering communications in urban and industrial environments is a common problem, and the existing technologies do not fully meet the increased requirements for the accuracy of working out the specified trajectories and efficiency of wells development. Laying engineering communications through water barriers is a special issue. The proposed method is based on the prompt installation of the forward end of the whipstock barrel in the required position for controlled correction of the trajectory of the well being laid. The main disadvantage of existing methods is the use of only passive trajectory control of the working body and the absence of control systems for the main processes of laying wells. The proposed system includes a guided barrel, a computerized control and management system being the basis for developing a technology for laying directional wells of small diameter and complex profile. The considered technology is the simplest and most economically profitable in comparison with drilling and punching. The possibility of using the described developments including for the purpose of directional drilling has been substantiated.
\end{abstract}

\section{Introduction}

The issues of laying engineering horizontal inclined wells intended both for exploration drilling and for laying engineering communications using the static puncture method as well as power cables and communication lines, arranging new ones and replacing worn out communications arise quite often. Water barriers, navigable canals, port waters are a fairly common problem when laying engineering communications and communication lines in coastal zones, in the basins of large rivers.

\section{Problem setting}

Horizontal directional drilling is the most modern and widespread method. Guided whipstocks are used for drilling multilateral wells and cross-sectional traces, vertical drilling, and today they are also adapted for the conditions of highly deviated and horizontal wells. They are a great way to ensure getting over water obstacles up to $600 \mathrm{~m}$ wide. Usually, communications are laid in these cases approximately 10 meters below the bottom. The disadvantage of this method is the high cost of work.

The most economical method is static well puncture. The limitation of this method until recently was the presence of only passive methods for correcting the coordinates of the well being laid. At present, guided barrels are used as an actuating element in such installations, which enables to significantly increase the accuracy of well drilling and, accordingly, increase their length. Static puncture is possible in soils of categories $1-4$.

In Russia the rate of engineering communications wearing out is quite high, which only increases the demand for their laying by a trenchless method. There is a need to replace worn out communications in industrial zones and areas of dense development. Requirements for the quality and pace of well construction are constantly growing, which makes the requirements for the accuracy of a given well trajectory more stringent and necessitates the use of only controlled methods of laying communications.

The choice of a method for laying communications through water barriers largely depend on the actual financial and technological capabilities but there are also situations when it is impossible to ensure the safety of the cable (pipeline) in any other way except for drilling or a controlled static puncture.

Guiding documents and current construction norms and specifications were developed decades ago, do not take into account current trends in the development of trenchless technologies and are limited to general recommendations for their choice, do not have methods for selecting equipment in specific construction conditions, do not contribute to ensuring the specified efficiency and compliance with safety requirements.

\footnotetext{
*Corresponding author: menjshenin@iwtsedov.ru
} 


\section{Description of design and operational concept of guided barrel}

Improvement of static puncture installations aims to increase the accuracy of laying underground communications by supplying a guided whipstock barrel with a mechanism for quick setting its tip in the required position in combination with active control of the path of guided whipstock barrel elements [1, 2, 3]. The route direction in the proposed device is corrected at the time of drilling the well due to the rotating working body, presented in the form of $[1,4]$ asymmetric leading tip, whose axis is deflected from the longitudinal axis of the tool. In this case, the position of the leading tip (working body) is controlled relative to the longitudinal axis of the barrel, and the position of the whipstock axis - relative to the vertical one regardless of the possible rotation of the stave being a measuring base.

The kinematic and functional diagrams of the developed guided whipstock barrel are presented in Figure 1.

The actuating (working) element of the controlled guided whipstock barrel is presented in the form of a link 1 (actuating cone) connected by means of a cardan joint 2 to the shaft 3 and link 4 made in the form of a bevel washer. These links rotate with different angular velocities in the same direction, which enables to reorient the actuating element and the entire guided whipstock barrel during its further advance into the wellhead. Link 4 is connected to a hollow cylinder 5, which receives rotation from an electric motor 8 by means of a gear transmission 6 and a gearbox 7. The entire structure of the guided whipstock barrel is assembled in the housing 9 of the first section of the guided whipstock barrel. Link 1 receives torque from the same electric motor 8 by means of the cardan joint 2 and the central shaft 3 connected to it, which is actually the output shaft of the gearbox 7 .

Such a constructive solution provides a fixed difference in the angular speeds of rotation of links 1 and 4 regardless of the speed of the built-in electric motor 8 . In the first section of the guided whipstock barrel there is a monitoring and control system. The issues of the kinematics of this mechanism are resolved with the objective to control the position of the working body. Engine speed control system is built into the engine of the guided whipstock barrel. The purpose of the second section of the guided whipstock barrel 10 is to axially advance the barrel in the wellhead by means of an electric motor 11 with a power jack block 12 .

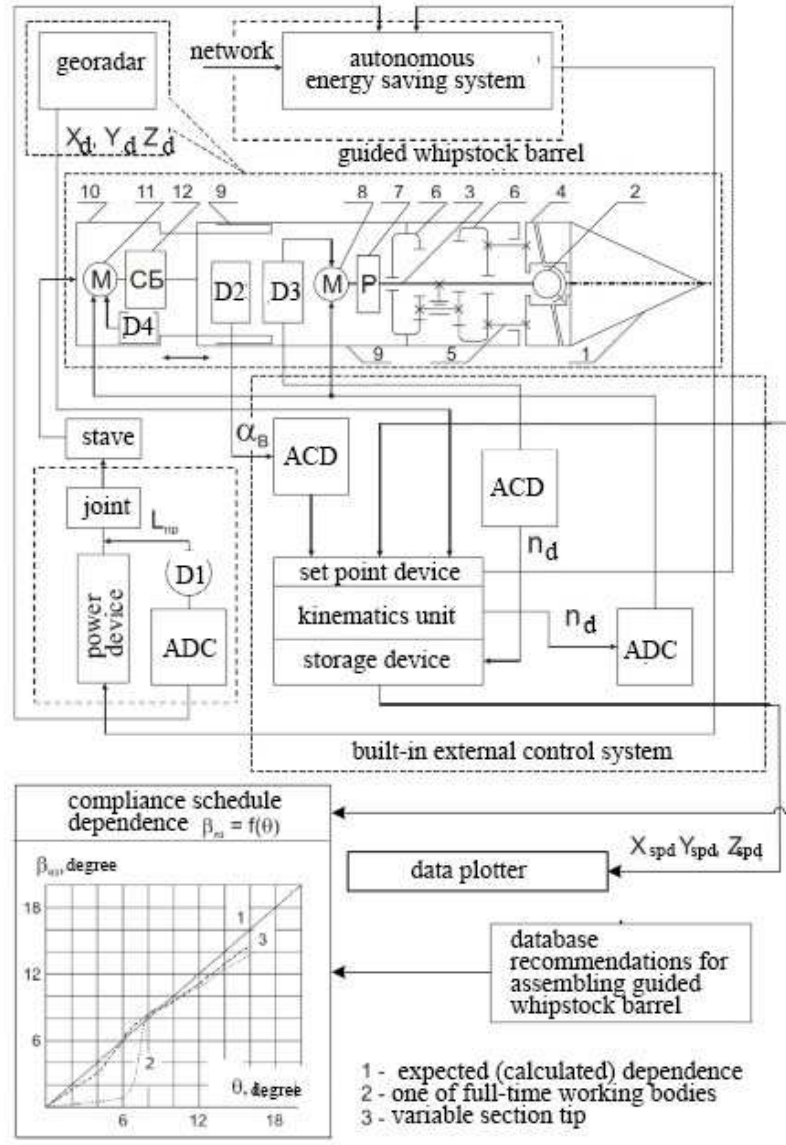

Fig. 1. Kinematic diagram of guided whipstock barrel and functional diagram representing installation of directional laying of engineering communications.

1 - output link; 2 - cardan joint; 3 - central shaft; 4 deflecting link; 5 - cage; 6 - gear transmission; 7 - reducer; 8 , 11 - electric motor; 9 -body of the first section; 10 -body of the second section; 11 -body of the second section; 12 - power unit; D1 - pump shutdown sensor; D2 - vertical position sensor; D3 - engine speed sensor; D4 - power unit engine speed sensor; $\Sigma$ - adder; ADC - analog-to-digital converter; $\mathrm{ACD}$ - analog converting device

\section{Description of technology for laying directional wells}

The technology for laying wells using a controlled geological survey is shown in Figure 1 and is as follows. Before starting work on laying a well, the physical and mechanical properties of the soil in the area of the trenchless passage are studied. Based on the data obtained, a project of the future route is created with an indication of straight sections and wellhead curvature radiuses. The project on laying engineering communications is processed in the database, in which the dependence of the angular value $\beta_{\text {curv }}$ (the angle formed by the axes of the adjacent sections of the stave when passing curvilinear sections) on the angular value $\theta$ (the value of the angle formed by the axes of rotation of links 1 and 4 of the guided whipstock barrel while reorienting). This enables to determine the control action on the working body of the guided whipstock barrel in the form of the number of revolutions $n_{D}$ of the electric 
motor 8 for each step of stave advancing into the ground in order to pass the curved sections of the projected route. The processed information in the database provides recommendations on the choice of the form of the guided whipstock barrel's actuator depending on the soils being developed, the configuration of the route and the conditions of its passage, stave rigidity, etc. The processed information from the database enters the control system of the guided whipstock barrel where it is represented as control signals.

The working and receiving pits are prepared. A power jack is installed in the working pit. The first section of the drilling rod with the attached guided whipstock barrel is attached to the lock of the working pit. The guided whipstock barrel is connected to the power supply and, if necessary, to an external system implementing monitoring and control over the position of the guided whipstock barrel. Power cables and control circuits run in the inner cavity of the drill string, which also serves as protection from external influences [4, 5].

The next technological stage is testing the monitoring and control systems of the guided whipstock barrel, as well as setting the initial coordinates of the position of the guided whipstock barrel and their compliance with the power plant position. The information is entered into the built-in control system. To control the position of the barrel in the process of laying the well, the following sensors are provided [1]: displacement sensor - D1 (controls the $\mathrm{L}_{\text {curv }}$ advancement); vertical sensor - D2 (determines $\alpha_{\mathrm{v}}$ being the angle of rotation of the guided whipstock barrel relative to the vertical axis, which is the measuring base); engine speed sensor SB - D4 (fixes the value of $n_{D S B}$ ); engine speed sensor of guided whipstock barrel - D3 (fixes the value of $n_{D}$ ).

Database enables to significantly reduce the probability of deviation of the obtained trajectory of the guided whipstock barrel from the design route. However, it is envisaged to use an external georadar (if possible) for additional control over the coordinates of the barrel's position during its operation. At certain fixed points of the route it helps to measure the actual position of the guided whipstock barrel at the wellhead of the well being developed; the obtained coordinate mismatch is recalculated by the unit for calculating the kinematics, aligned with the database recommendations, and fed to the electric motor 8 as a value of $n_{D}$.

An electric motor 8 built into the guided whipstock barrel transmits the torque to the output link 1 of the working body by means of a gearbox 7 , a central shaft 3 and a cardan joint 2. The second link of the working body being the swash plate 4 made integrally with the hollow cylinder 5 rotates from the electric motor 8 by means of the central shaft 3 and additional gear 6 . As a result, the links 1 and 4 of the working body of the guided whipstock barrel rotate relative to each other with different angular velocities, reorient the position of the working body relative to the axis of the guided whipstock barrel body, thus ensuring the prompt installation of the working body of the guided whipstock barrel in the required position relative to the axis in coaxial relationship as well.
Next, the process of direct well drilling, which is divided into advancement stages associated with the long stroke of the rods of the power hydraulic jack, begins.

Operations are performed in the following sequence.

Stage 1. The required position of the working body of the guided whipstock barrel is set.

The power unit built into the second section of the guided whipstock barrel implements the first section into the soil mass. Its advancement step is determined by the number of revolutions $\mathrm{n}_{\mathrm{DSB}}$ of the motor shaft 11 and is monitored by the engine speed sensor $\mathrm{D} 4$. The operation is carried out for the entire stroke of the lead screw of the power unit.

Stage 2. At the same time, the external jacking power plant and the built-in power unit are switched on. The built-in power unit works in reverse by shifting the two sections of the guided whipstock barrel to its original position, and the power jack stops the working body from moving backwards by creating a certain supporting force. To carry out this process, the operating speeds of the power plants are synchronized and controlled by sensors D4 and D1. This stage ends with the complete closure of the two sections of the guided whipstock barrel.

Step 3. The lock on the external power unit is released and the drill string gets free. The cylinder rods of the power plant move to the initial position and the lock is fixed on the stave. If necessary, the next section of the drillstring is added.

Stage 4 . In parallel with stage 3 , the monitoring and control system determines the coordinates of the guided whipstock barrel at the wellhead $\left(\mathrm{X}_{\mathrm{i}}, \mathrm{Y}_{\mathrm{i}}, \mathrm{Z}_{\mathrm{i}}\right)$, which are compared with its design values $\left(\mathrm{X}_{\text {set }}, \mathrm{Y}_{\text {set }}, \mathrm{Z}_{\text {set }}\right)$. Based on these data, an error signal fed to the control system of the guided whipstock barrel $\left(\mathrm{n}_{\mathrm{D}}\right.$ for engine 8 ) is generated. This value is determined by the block for calculating kinematics compared with the database (dependence $\beta_{\text {curv }}=\mathrm{f}(\theta)$ and corrected taking into account the readings of the vertical sensor D2 and the engine speed sensor D3. Thus, a new control action is calculated on the guided whipstock barrel with simultaneous control of the accuracy of the specified coordinates of the guided whipstock barrel at the wellhead. At the same stage (if necessary), the control over processing of the specified coordinates by the georadar is carried out. The combination of stages 3 and 4 can significantly speed up the well development as a whole.

Further, these stages are repeated in the same sequence until the guided whipstock barrel enters the receiving pit. Here the guided whipstock barrel is dismantled. Its place is taken, for example, by a well reamer, which will increase the diameter of the developed well during the stave return stroke. In some cases, the drill string can be left in the well and serve as a kind of protection for the power cable line or fiber optic communication, etc. [4, 5]. 


\section{Model of barrel-ground interaction}

The developed model is based on the description of the distribution of pressure profiles during the introduction of a round die, which changes from a parabola to a saddle-shaped curve with an increase in the degree of soil cohesion. The compaction core is formed in the zones of stamp sole action and of side surfaces action. It is also taken into account that the static and dynamic characteristics of the penetration of the tool coincide at low speeds (up to $30 \mathrm{~m} / \mathrm{s}$ ).

The authors carried out experimental studies on a barrel model to study the interaction of tips (tools) with different geometrical parameters and soils. The research program included experiments with various physical and mechanical properties of soils. As a result, the dependence of the magnitude of the soil resistance forces to penetration on the length of the lateral surface of the inserted tip, which in turn determines the area of the contact surface of the compaction core formed during the interaction of the tool with the soil, has been substantiated. When the tip is placed in an eccentric position, the difference in the size of opposite sides of the contact in the plane of eccentricity formation can be described by the following formula (Figure 2):

$$
L_{i}=\frac{2 R_{\operatorname{con}_{i}}\left(\cos \frac{\alpha_{\kappa}}{2} \pm \sin \frac{\alpha_{\kappa}}{2} \operatorname{tg} \beta_{\kappa}\right)}{\sin \alpha_{\kappa}\left(\frac{2}{1+\cos 2 \beta_{\kappa}}+\operatorname{tg} \beta_{\kappa}\left(\operatorname{tg} \frac{\alpha_{\kappa}}{2}-\operatorname{tg} \beta_{\kappa}\right)\right)}
$$

where $R_{\text {con }_{i}}$ is a tool radius in a plane perpendicular to its axis of rotation at a feed rate $\mathrm{i}, \mathrm{mm} ; \mathrm{L}_{\mathrm{i}}$ is the length of the lateral surface of the penetrated tool at the same feed rate $i, \mathrm{~mm} ; \beta_{\mathrm{K}}$ is an angle of deviation of the axis of tool rotation from the normal to the soil surface, degree; $\alpha_{\mathrm{K}}$ is a tool tip angle, degree.

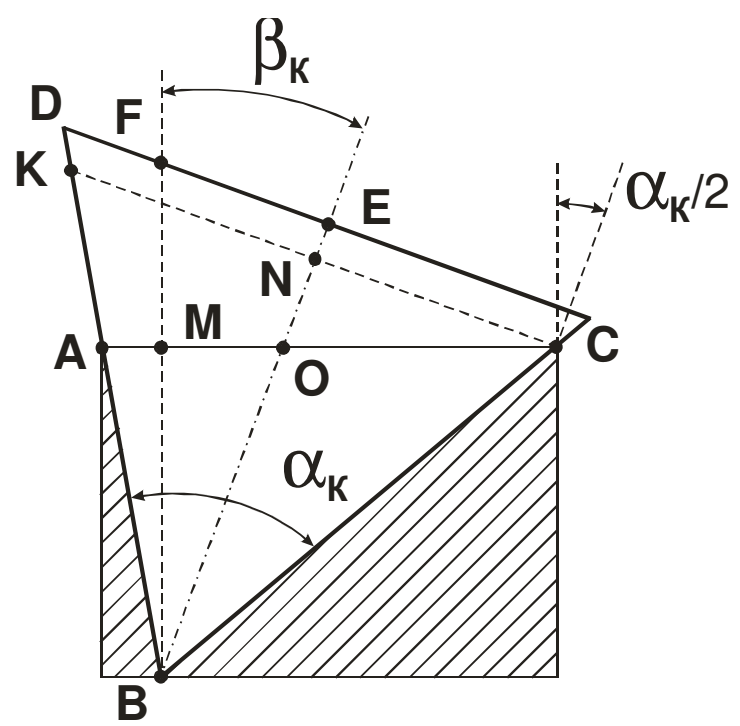

Fig. 2. Design scheme for determining volume of displaced and compacted soil when introducing tool with eccentricity

As a result, even with an insignificant eccentricity, forces leading to a curvature of the borehole axis and depending on the ratio of the lengths of the tool's opposite lateral surfaces in the plane of the eccentricity manifestation are formed.

The axial penetration force $R_{o s}$ is related to the volume of the displaced soil $\mathrm{V}_{\mathrm{gr}}$ according to a linear relationship that can be determined for each standard size of the tool depending on the angle $\alpha_{\mathrm{K}}$ at the top and the area of the base of the cone $S_{\text {con }}$ (Figure 3 ).

The dependence of the axial force on the geometric parameters of the inserted tips and the physical and mechanical properties of the traversed soils were substantiated in the course of the following experiment.

For the study, the tips of various shapes but of the same height $\mathrm{H}$ equal to $90 \mathrm{~mm}$ were applied. They were introduced into the soil with certain physical and mechanical properties to a control depth equal to the twofold height $(180 \mathrm{~mm})$ and the axial penetration force $\mathrm{R}_{\mathrm{os}}$ was measured. For the study, we used soil with natural compaction after removing the upper layer.

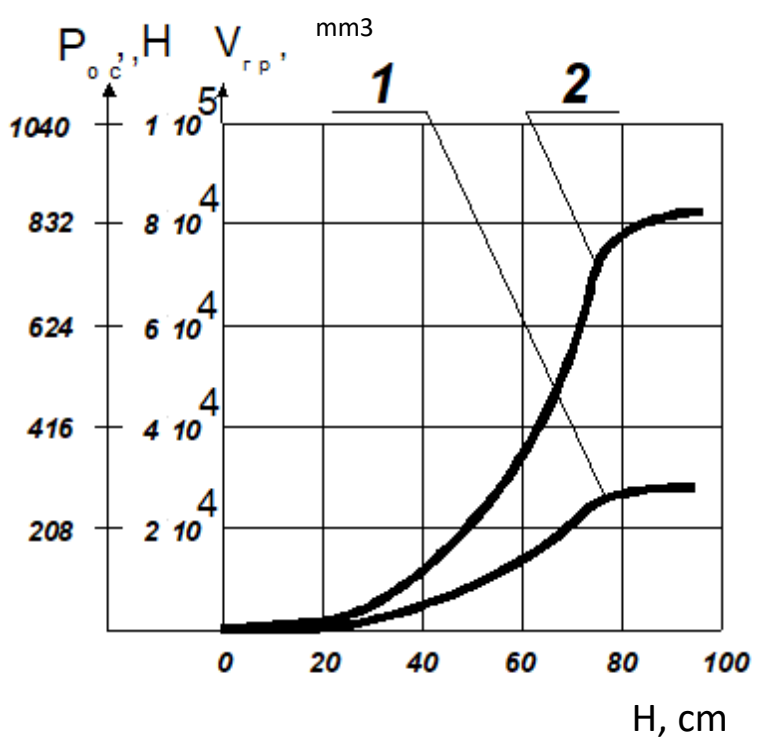

Fig. 3. Dependence of axial force of $\mathrm{R}_{\mathrm{os}}$ implementation, volume of displaced and compacted soil $\mathrm{V}_{\text {os }}$ when inserting a tool with an eccentricity from the penetration depth $\mathrm{H}$ (according to Fig. 2.)

1 is a graph of dependence on the surface of the BD tool,

2 is a graph of dependence on the surface of the $\mathrm{BC}$ tool

A rapid increase in the axial force $R_{o s}$ during well laying occurs at the first stage of penetration to a depth equal to $2 \mathrm{~N}$, which is explained by the formation of a compaction core [5].

The axial force of a static puncture introduction for the given conditions for laying wells is determined by:

$$
P_{o s}=\frac{\pi R_{\text {well }}^{2} \delta_{\text {comp }}}{U_{o}}+q_{m} L f g,
$$

where $\mathrm{R}_{\text {well }}$ is well section radius, $\mathrm{cm}$,

$\delta_{\text {comp }}$ is coefficient of soil resistance to compaction, MPa, $\mathrm{U}_{\mathrm{O}}$ is soil porosity before puncture,

$\mathrm{Q}_{\mathrm{T}}$ is weight of 1 meter of the laid stave, $\mathrm{kg}$,

$\mathrm{L}$ is penetration length, $\mathrm{m}$,

$\mathrm{f}$ is coefficient of friction of steel on soil.

Thus, the axial force is proportional to the borehole penetration length not taking into account the additional 
energy consumption for the formation of the compaction core.

Experimental studies prove that $R_{o s}$ is directly dependent on the volume of the displaced and compacted soil and the area of the side surface of the tool, the friction surface $S_{\text {lat }}$ or the diameter of the whipstock $-\mathrm{D}$ :

$$
P_{o s}=V_{g r} \rho_{g r} 10^{6} g+S_{l a t} \delta_{\text {comp }} f, \mathrm{H},
$$

where $\mathrm{V}_{\mathrm{gr}}$ is the volume of compacted and displaced soil, $\mathrm{mm}^{3}$;

$\rho_{\mathrm{gr}}$ is density of the traversed soil, $\mathrm{g} / \mathrm{cm}^{3}$;

$\mathrm{g}$ is acceleration of gravity, $\mathrm{m} / \mathrm{s}^{2}$;

$S_{\text {lat }}$ is the area of the lateral surface of the tool, friction surface, $\mathrm{mm}^{2}$;

$\mathrm{f}$ is the coefficient of friction of steel on the ground;

$\delta_{\text {comp }}$ is coefficient of soil resistance to compaction, MPa.

The volume of the total compacted and displaced soil $\mathrm{V}_{\mathrm{gr}}$ when driving a well to a depth of $2 \mathrm{H}$ is as follows:

$$
V_{g r}=\frac{4}{3} \pi R_{\text {well }}^{2} H,\left[\mathrm{~mm}^{3}\right],
$$

where $R_{w e l l}^{2}$ is a borehole radius equal to the tip base diameter $\mathrm{D}, \mathrm{mm}$.

Lateral surface area is as follows:

$$
S_{\text {lat }}=\frac{\pi H D}{2 \cos \frac{\alpha}{2}},\left[\mathrm{~mm}^{3}\right]
$$

When processing the above equations, the equation for determining $\mathrm{P}_{\mathrm{os}}$ takes the following form:

$$
P_{\text {os }}=S_{\text {base }} H\left[\frac{4}{3} \rho g 10^{6}+2 \frac{\delta_{\text {comp }} f}{D \cos \frac{\alpha}{2}}\right] \text {, }
$$

where $S_{\text {base }}$ is the area of the base of the cone being introduced or the cross-sectional area of the well, $\mathrm{mm}^{2}$.

Thus, the axial force of the cone penetration into the soil is proportional to the weight of the displaced soil in the volume of the tool used and is formed due to compaction taking into account friction losses.

The discrepancies between the actual and calculated values of $R_{o s}$ fluctuate within $15-20 \%$, which is within the permissible experimental error. The dependence $R_{o s}$ $=\mathrm{f}(\mathrm{H})$ (after deepening the well by more than $2 \mathrm{~N}$ ) is straight-line with a small degree of increase in the axial force compared to the length of the well penetration.

\section{Main findings and conclusion}

A significant characteristic of the proposed solution is the division of the power effort into two components (the presence of two power plants). The internal power plant operates with a constant force determined by the resistance of the soil massif to the movement of the guided whipstock barrel. Such a solution significantly reduces the efforts on the external power plant, which constantly increases with the long penetration since it is required to overcome the frictional forces of the steel against the walls of the well [1] especially in curved sections. The transfer of the efforts of penetration directly into the well increases the accuracy of the given trajectory since the efforts are transferred practically to the actuating element of the guided whipstock barrel without taking into account the constantly increasing length of the stave. Consequently, there emerges a possibility to increase the possible well length without increasing the capacity of the power plant and losing the accuracy of a given trajectory. The bending strain acting on the drill string is reduced, which reduces the likelihood of their direct contact with the borehole walls and increases the overall trouble-free operation.

It should be noted that the developed functional and constructive solutions are universal, adapted to the conditions of not only puncture and punching but also drilling of horizontal wells.

\section{References}

1. A. N. Drovnikov, S. E. Menshenin, E. A. Menshenina, 2017 International Conference on Industrial Engineering, Applications and Manufacturing (ICIEAM), pp. 1-4, (2017)

2. B. B. Danilov, A. S. Kondratenko, B. M. Smolyanitsky, A. S. Smolentsev, J Min. Sci., 53 (3), pp 478-483 DOI: 10.1134/S1062739117032391

3. Weibo L 2008 Construction Technology Retrieved from: en.cnki.com.cn

4. F. Matsumoto, T. Morita, E. Sakai, H. Shimada, T. Sasaoka, K. Matsuii, International Conference and Exibition No-Dig 2013. Paper 3-4, Sydney, 1-4 September 2013, s.1-7

5. T. Bok, A. Drovnikov, J. Popkov, S. Menshenin 21 international symposium on automatics and robototechnics in construction. Seul, pp 46-50. (2004). 